\section{Assessment of medical technology}

The Image and the Reality: A Case Study of the Impacts of Medical Technology. By B. Stocking and S. L. Morrison. Pp. 80. (Oxford University Press: Oxford, London and New York, 1978.) Paperback $£ 3$.

THERE is no doubt about the public appeal of medical technology, as the achievement of a remarkable lady in Lancashire is raising nearly $£ 1.5$ million to buy and run a body scanner attests. Yet as the Director-General of WHO has recently said: "Technology for the sake of technology is a dangerous addiction-producing drug". Stocking and Morrison tackle the problems of technology assessment taking the body scanner as a case study.

The development of computed tomographic (CT) scanning of the brain by EMI, funded by the Department of Health and Social Security, was a remarkable British success story leading to clear improvements in diagnosis. The same technique-X-rays passed through the body, detected by scintillation counters coupled to a computer-was then extended to whole body scanning. Whereas clinical evaluation had accompanied development of the brain scanner, in the case of whole body scanners evaluation was more complex because of the variety of organs involved and of alternative diagnostic techniques and they were extensively introduced before there had been thorough clinical evaluation. The most rapid diffusion of body scanners has taken place in the USA (700 machines by 1977) and is largely attributable to their profitability, whereas in Britain diffusion has been much slower (11 machines by 1977) and for most of these the capital, but not running, costs have been met by charities rather than the health service.

The impact of body scanners is considered in this book on three broad levels: for patients, in terms of diagnosis, patient management and outcome; for the health service, in terms of the pattern and demand for care and for the professions involved; and for industry involved in manufacturing the equipment. As assessment of any technology must be concerned with the future, the authors discuss a variety of changes that may affect the contribution of CT scanners such as future developments of CT scanners, advances in other imaging techniques and in therapy, changes in cost and health service expenditure. This of course introduces many uncertainties but it is surely wise to try and see what may develop and the implications; as decisions affect the future it makes little sense to base them solely on an evaluation of the past.

In assessing medical technology, what is ultimately most important is the effect on outcome for the patient. Randomised controlled trials are common, if far from universal, practice in evaluating new drugs and therapies -however unusual such trials may be in assessing accepted techniques and therapies - and there is every reason to extend them to diagnostic procedures. Yet this is only just beginning.

Stocking and Morrison write: "The remarkable diffusion of [whole body scanners] prior to proper clinical evaluation is unsatisfactory". This is perhaps an understatement. The image of the health service promoted by the medical profession in Britain and many other countries is that it is crumbling from lack of resources and deprived of new developments that could be saving lives. What may be closer to the reality is that much of the medical profession is quite unscientific, indifferent to clinical evaluation and infatuated with technology and that there are ample resources if only they can be

\section{Testicular function}

The Mammalian Testis. By R. B. Setchell. Pp. 450. (Paul Elek: London, 1978.) $£ 18$.

THE testis is responsible for the production of spermatozoa and testicular hormones. Highlights of spermatozoa production include the initial mitotic divisions, the setting aside of stem cells which will re-initiate the spermatogenic process at some later date, meiosis, and finally, the dramatic metamorphosis of the prospective gamete that results in the formation of the acrosome, flagellum and mitochondrial helix and in the condensation of nuclear chromatin. Interestingly, many of these events occur only if the prospective gamete is sequestered behind the blood-testis barrier formed by the Sertoli-Sertoli occluding junctions bathed in fluids rich in steroid hormones and protected from noxious stimuli such as elevated temperature and radiation.

Dr Setchell's book deals with all the above aspects of testicular function. This is a formidable task because it requires that the author be knowledgeable in anatomy, physiology, bio- re-directed from activities which are useless and downright harmful into those where they may do some good.

This slim volume is interesting as a case study, and one can only lament that it is the first such study to approach a new technology in a systematic and comprehensive way. It is still more valuable in that it poses fundamental questions about the nature of health services and the impact of technology on these-which is not to doubt the highly beneficial effects in many cases. Finally, the authors make a positive proposal for a Medical Technology Assessment Group. With the prospect of almost limitless extensions of what can be done with medical technology, decisions as to what should be done can only become more pressing. The sooner Departments of Health and the World Health Organisation regard the assessment of medical technology as central to their functions the more hope there is of shaping health services to conform with the needs of human life.

David Piachaud

David Piachaud is Lecturer in Social Administration at the London School of Economics and Political Science.

chemistry and endocrinology. For the most part, Dr Setchell achieves this difficult task with the result that the book is informative, clearly written, well illustrated and completely referenced. It will provide a valuable source of information for all those interested in testicular structure and function.

The chapters on vascular and nervous supply of the testis and scrotum, on fluid secretion and entry of substances into the seminiferous tubules, and on testicular thermoregulation are extremely good and reflect the author's long-standing research interest and expertise on these subjects. The chapters on spermatogenesis, endocrinology of the testis and endocrine control of the testis are presented in a thorough and professional manner. The chapters dealing with testicular metabolism and naturally occurring and induced dysfunctions of the testis are informative but not insightful. They consist primarily of a recitation of literature references and contain little or no synthesis of concepts. Overall, this is an excellent book and Dr Setchell should be congratulated.

Larry Ewing

Larry Ewing is Professor and Head of the Division of Reproductive Biology in the Department of Population Dynamics, Johns Hopkins School of Hygiene and Public Health, Baltimore, Maryland. 